\title{
LITTLEWOOD-PALEY THEOREM FOR SCHRÖDINGER OPERATORS
}

\author{
SHIJUN ZHENG
}

\begin{abstract}
Let $H$ be a Schrödinger operator on $\mathbb{R}^{n}$. Under a polynomial decay condition for the kernel of its spectral operator, we show that the Besov spaces and Triebel-Lizorkin spaces associated with $H$ are well defined. We further give a Littlewood-Paley characterization of $L_{p}$ spaces as well as Sobolev spaces in terms of dyadic functions of $H$. This generalizes and strengthens the previous result when the heat kernel of $H$ satisfies certain upper Gaussian bound.
\end{abstract}

\section{INTRODUCTION AND MAIN RESULTS}

Recently the theory of function spaces associated with Schrödinger operators have been drawing attention in the area of harmonic analysis and PDEs [12, 2, 1, 14, 16, 9, 6, 8, 1, 15. In 9, 6, 1, 14, it is proved that the Besov and Trieble-Lizorkin spaces associated with a Schrödinger operator are well defined, in some particular cases. In this note we aim to extend the result for general Schrödinger operators on $\mathbb{R}^{n}$. Furthermore we are interested in obtaining a Littlewood-Paley decomposition for the $L_{p}$ spaces as well as Sobolev spaces using dyadic functions of $H$.

Let $H=-\Delta+V$ be a Schrödinger operator that is selfadjoint in $L_{2}\left(\mathbb{R}^{n}\right)$ with a real-valued potential function $V$. Then for a Borel measurable function $\phi$, one can define the spectral operator $\phi(H)$ by functional calculus $\phi(H)=\int_{-\infty}^{\infty} \phi(\lambda) d E_{\lambda}$, where $d E_{\lambda}$ is the spectral measure of $H$. The kernel of $\phi(H)$ is denoted $\phi(H)(x, y)$.

Let $\left\{\varphi_{j}\right\}_{j \in \mathbb{Z}} \subset C_{0}^{\infty}(\mathbb{R})$ be a smooth dyadic system satisfying the conditions (i) $\operatorname{supp} \varphi_{j} \subset\left\{x: 2^{j-2} \leq|x| \leq 2^{j}\right\}$

Date: July 26, 2006.

2000 Mathematics Subject Classification. Primary: 42B25; Secondary: 35P25.

Key words and phrases. functional calculus, Schrödinger operator, LittlewoodPaley theory.

This work is supported by DARPA grant HM1582-05-2-0001. The author gratefully thanks the hospitality and support of Department of Mathematics, University of South Carolina, during his visiting at the Industrial Mathematics Institute. 
(ii) $\left|\varphi_{j}^{(k)}(x)\right| \leq c_{k} 2^{-k j}, \quad \forall j \in \mathbb{Z}, k \in \mathbb{N}_{0}=\{0\} \cup \mathbb{N}$

(iii)

$$
\sum_{j=-\infty}^{\infty} \varphi_{j}(x) \approx c>0, \quad \forall x \neq 0 .
$$

Let $0<p<\infty, 0<q \leq \infty$ and $\alpha \in \mathbb{R}$. The homogenous TriebelLizorkin space $\dot{F}_{p}^{\alpha, q}(H)$ is defined as the completion of the Schwartz class $\mathcal{S}\left(\mathbb{R}^{n}\right)$ with the quasi-norm

$$
\|f\|_{\dot{F}_{p}^{\alpha, q}(H)}=\left\|\left(\sum_{j=-\infty}^{\infty} 2^{j \alpha q}\left|\varphi_{j}(H) f(\cdot)\right|^{q}\right)^{1 / q}\right\|_{p} .
$$

Similarly, if $0<p \leq \infty, 0<q \leq \infty$, the homogeneous Besov space $\dot{B}_{p}^{\alpha, q}(H)$ is defined by the quasi-norm

$$
\|f\|_{\dot{B}_{p}^{\alpha, q}(H)}=\left(\sum_{j=-\infty}^{\infty} 2^{j \alpha q}\left\|\varphi_{j}(H) f\right\|_{p}^{q}\right)^{1 / q} .
$$

Throughout this note we assume $H$ satisfies the following:

Assumption 1.1. Let $\phi_{j} \in C_{0}^{\infty}(\mathbb{R})$ be as in condition (i), (ii). Then for every $N \in \mathbb{N}_{0}$ there exists a constant $c_{N}>0$ such that for all $j \in \mathbb{Z}$

$$
\begin{gathered}
\left|\phi_{j}(H)(x, y)\right| \leq c_{N} \frac{2^{n j / 2}}{\left(1+2^{j / 2}|x-y|\right)^{N}} \\
\left|\nabla_{x} \phi_{j}(H)(x, y)\right| \leq c_{N} \frac{2^{(n+1) j / 2}}{\left(1+2^{j / 2}|x-y|\right)^{N}} .
\end{gathered}
$$

This is the case when $H$ is the Hermite operator $-\Delta+|x|^{2}$, or more generally, whenever $V$ is nonnegative and $H$ satisfies the upper Gaussian bound for the heat kernel and its derivative (see Proposition 3.3. $)$. However, when the potential $V$ is negative, such a heat kernel estimate is not available. Therefore it is necessary to consider a more general condition as given in Assumption 1.1

Define the Peetre maximal function for $H$ as: for $j \in \mathbb{Z}, s>0$

$$
\varphi_{j, s}^{*} f(x)=\sup _{t \in \mathbb{R}^{n}} \frac{\left|\varphi_{j}(H) f(t)\right|}{\left(1+2^{j / 2}|x-t|\right)^{s}},
$$

and

$$
\varphi_{j, s}^{* *} f(x)=\sup _{t \in \mathbb{R}^{n}} \frac{\left|\left(\nabla_{t} \varphi_{j}(H) f\right)(t)\right|}{\left(1+2^{j / 2}|x-t|\right)^{s}} .
$$


The following theorem is a maximal characterization of the homogeneous spaces. By $\|\cdot\|_{A} \approx\|\cdot\|_{B}$ we mean equivalent norms.

Theorem 1.2. Suppose $H$ satisfies Assumption [1.1.

a) If $0<p \leq \infty, 0<q \leq \infty, \alpha \in \mathbb{R}$ and $s>n / p$, then

$$
\|f\|_{\dot{B}_{p}^{\alpha, q}(H)} \approx\left\|\left\{2^{j \alpha} \varphi_{j, s}^{*}(H) f\right\}\right\|_{\ell^{q}\left(L_{p}\right)} .
$$

b) If $0<p<\infty, 0<q \leq \infty, \alpha \in \mathbb{R}$ and $s>n / \min (p, q)$, then

$$
\|f\|_{\dot{F}_{p}^{\alpha, q}(H)} \approx\left\|\left\{2^{j \alpha} \varphi_{j, s}^{*}(H) f\right\}\right\|_{L_{p}\left(\ell^{q}\right)} .
$$

It is well-known that such a characterization implies that any two dyadic systems satisfying (i), (ii), (iii) give rise to equivalent norms

on $\dot{F}_{p}^{\alpha, q}(H)$ and $\dot{B}_{p}^{\alpha, q}(H)$. The analogous result also holds for the inhomogenous spaces $F_{p}^{\alpha, q}(H), B_{p}^{\alpha, q}(H)$. However, the homogeneous spaces, which cover both high and low energy portion of $H$, are essential and more useful in proving Strichartz inequality for wave equations [16, 13. This is one reason of our motivation.

Following the same idea in [14, using Calderón-Zygmund decomposition and Assumption 1.1 we show that $L_{p}\left(\mathbb{R}^{n}\right)=F_{p}^{0,2}(H)$ if $1<p<\infty$. We thus obtain the Littlewood-Paley theorem for $L_{p}$ spaces.

Theorem 1.3. Suppose $H$ satisfies Assumption 1.1. If $1<p<\infty$, then

$$
\|f\|_{L_{p}\left(\mathbb{R}^{n}\right)} \approx\left\|\left(\sum_{j=-\infty}^{\infty}\left|\varphi_{j}(H) f(\cdot)\right|^{2}\right)^{1 / 2}\right\|_{L_{p}\left(\mathbb{R}^{n}\right)} .
$$

Under additional condtion on $V$, e.g., $\left|\partial_{x}^{k} V(x)\right| \leq c_{k},|k| \leq 2 m_{0}-2$ for some $m_{0} \in \mathbb{N}$, we can characterize the Sobolev spaces $H_{p}^{2 s}\left(\mathbb{R}^{n}\right)=$ $F_{p}^{s, 2}(H), 1<p<\infty,|s| \leq m_{0}$ with equivalent norms

$$
\|f\|_{H_{p}^{2 s}\left(\mathbb{R}^{n}\right)} \approx\left\|\left(\sum_{j=-\infty}^{\infty} 2^{2 j s}\left|\varphi_{j}(H) f(\cdot)\right|^{2}\right)^{1 / 2}\right\|_{L_{p}\left(\mathbb{R}^{n}\right)} .
$$

\section{Proofs of Theorem 1.2 and Theorem 1.3}

The proof of Theorem 1.2 is standard and follows from Bernstein type inequality (Lemma 2.11) and Peetre type maximal inequality (Lemma 2.2) for maximal functions.

Lemma 2.1. For $s>0$, there exists a constant $c_{n, s}>0$ such that for all $j \in \mathbb{Z}$

$$
\varphi_{j, s}^{* *} f(x) \leq c_{n, s} 2^{j / 2} \varphi_{j, s}^{*} f(x), \quad \forall f \in \mathcal{S}\left(\mathbb{R}^{n}\right) .
$$


Similar to 20, 14 Lemma 2.1] can be easily proved using (21) with $N>n+s$ and the identity

$$
\varphi_{j}(H) f(x)=\psi_{j}(H) \varphi_{j}(H) f
$$

where $\psi_{j}(x)=\psi\left(2^{-j} x\right)$ with $\psi \in C_{0}^{\infty}$, supp $\psi \subset\left\{\frac{1}{5} \leq|x| \leq \frac{5}{4}\right\}$ and $\psi(x)=1$ on $\left\{\frac{1}{4} \leq|x| \leq 1\right\}$.

Let $M$ denote the Hardy-Littlewood maximal function

$$
M f(x)=\sup _{B \ni x} \frac{1}{|B|} \int_{B}|f(y)| d y
$$

where the supreme is taken over all balls $B$ in $\mathbb{R}^{n}$ centered at $x$.

Lemma 2.2. Let $0<r<\infty$ and $s=n / r$. Then for all $j \in \mathbb{Z}$

$$
\varphi_{j, s}^{*} f(x) \leq c_{n, r}\left[M\left(\left|\varphi_{j}(H) f\right|^{r}\right)\right]^{1 / r}(x), \quad \forall f \in \mathcal{S}\left(\mathbb{R}^{n}\right) .
$$

Proof. Let $g(x) \in C^{1}\left(\mathbb{R}^{n}\right)$. As in [20, 1], the mean value theorem gives for $z_{0} \in \mathbb{R}^{n}, \delta>0$

$$
\left|g\left(z_{0}\right)\right| \leq \delta \sup _{\left|z-z_{0}\right| \leq \delta}|\nabla g(z)|+c_{n, r} \delta^{-n / r}\left(\int_{\left|z-z_{0}\right| \leq \delta}|g|^{r} d z\right)^{1 / r} .
$$

Put $g(z)=\varphi_{j}(H) f(x-z)$ to get

$$
\begin{aligned}
& \frac{\left|\varphi_{j}(H) f(x-z)\right|}{\left(1+2^{j / 2}|z|\right)^{n / r}} \leq \delta \sup _{|u-z| \leq \delta} \frac{\left(1+2^{j / 2}|u|\right)^{n / r}\left|\nabla\left(\varphi_{j}(H) f\right)(x-u)\right|}{\left(1+2^{j / 2}|z|\right)^{n / r}\left(1+2^{j / 2}|u|\right)^{n / r}} \\
+ & c_{n, r} \delta^{-n / r}\left(1+2^{j / 2}|z|\right)^{-n / r}\left(\int_{|u-z| \leq \delta}\left|\varphi_{j}(H) f(x-u)\right|^{r} d u\right)^{1 / r} \\
\leq & \delta\left(1+2^{j / 2} \delta\right)^{n / r} \varphi_{j, s}^{* *} f(x)+c_{n, r} \delta^{-n / r}\left(\frac{|z|+\delta}{1+2^{j / 2}|z|}\right)^{n / r}\left[M\left(\left|\varphi_{j}(H) f\right|^{r}\right)(x)\right]^{1 / r} \\
\leq & c_{n, r} \delta\left(1+2^{j / 2} \delta\right)^{n / r} 2^{j / 2} \varphi_{j, s}^{*} f(x)+c_{n, r} \delta^{-n / r}\left(\frac{|z|+\delta}{1+2^{j / 2}|z|}\right)^{n / r}\left[M\left(\left|\varphi_{j}(H) f\right|^{r}\right)\right]^{1 / r}(x) \\
\leq & c_{n, r} \epsilon(1+\epsilon)^{n / r} \varphi_{j, s}^{*} f(x)+c_{n, r}\left(1+\epsilon^{-1}\right)^{n / r}\left[M\left(\left|\varphi_{j}(H) f\right|^{r}\right)\right]^{1 / r}(x),
\end{aligned}
$$

by setting $\delta=2^{-j / 2} \epsilon, \epsilon>0$ and using Lemma 2.1. Finally, taking $\epsilon>0$ sufficiently small establishes (44).

Now Theorem 1.2 is a consequence of Lemma 2.2 and the following well-known lemma on Hardy-Littlewood maximal function by a standard argument; see [20] or [9, 14] for some simple details.

Lemma 2.3. a) If $1<p \leq \infty$, then

$$
\|M f\|_{L_{p}\left(\mathbb{R}^{n}\right)} \leq C_{p}\|f\|_{L_{p}\left(\mathbb{R}^{n}\right)}
$$


b) If $1<p<\infty, 1<q \leq \infty$, then

$$
\left\|\left(\sum_{j}\left|M f_{j}\right|^{q}\right)^{1 / q}\right\|_{L_{p}\left(\mathbb{R}^{n}\right)} \leq C_{p, q}\left\|\left(\sum_{j}\left|f_{j}\right|^{q}\right)^{1 / q}\right\|_{L_{p}\left(\mathbb{R}^{n}\right)} .
$$

2.4. Proof of Theorem 1.3. From the proof of the identification of $F_{p}^{0,2}(H)$ spaces [14, Theorem 5.1] we observe that the estimates in (11), (2) imply

$$
\|f\|_{F_{p}^{0,2}(H)} \approx\|f\|_{L_{p}}, \quad 1<p<\infty
$$

by applying $L_{p}\left(\ell^{2}\right)$-valued Calderón-Zygmund decomposition. On the other hand, Theorem 1.2 suggests that

$$
\|f\|_{F_{p}^{\alpha, q}(H)} \approx\left\|\left\{2^{j \alpha} \varphi_{j}(H) f\right\}\right\|_{L_{p}\left(\ell^{q}\right)}
$$

whenever $\left\{\varphi_{j}\right\}_{j \in \mathbb{Z}}$ is a dyadic system satisfying (i), (ii), (iii).

Combining (17) and (8) with $\alpha=0, q=2$ proves Theorem 1.3.

Remark 2.5. For $p=1$, Dziubański and Zienkiewicz [7] recently obtained a characterization of Hardy space associated with $H$ and showed that if a compactly supported positive potential $V$ is in $L^{n / 2+\epsilon}, n \geq 3$, then

$$
\|f\|_{\mathcal{H}^{1}} \approx\|w f\|_{H^{1}\left(\mathbb{R}^{d}\right)}
$$

where $\mathcal{H}^{1}=\left\{f \in L^{1}: \sup _{t>0}\left|e^{-t H} f(\cdot)\right| \in L^{1}\right\}$ and the weight $w$ is defined by $w(x)=\lim _{t \rightarrow \infty} \int_{\mathbb{R}^{n}} e^{-t H}(x, y) d y$. It would be very interesting to see whether one can give a Littlewood-Paley characterization of $\mathcal{H}^{1}$ in the sense of Theorem 1.3 .

\section{Potentials satisfying upper Gaussian Bound}

In this section we show that Assumption 1.1 is verified when $H$ satisfies the upper Gaussian bound (10) for its heat kernel. We begin with a weighted $L^{1}$ inequality, which is an easy consequence of [11, Lemma 8] by a scaling argument.

Lemma 3.1. (Hebisch) Suppose $V \geq 0$ and $e^{-t H}$ satisfies

$$
0 \leq e^{-t H}(x, y) \leq c_{n} t^{-n / 2} e^{-c|x-y|^{2} / t}, \quad \forall t>0 .
$$

If $s>(n+1) / 2+\beta, \beta \geq 0$ and supp $g \subset[-10,10]$, then

$$
\sup _{j \in \mathbb{Z}, y \in \mathbb{R}^{n}}\left\|g\left(2^{-j} H\right)(\cdot, y)\left\langle 2^{j / 2}(\cdot-y)\right\rangle^{\beta}\right\|_{L^{1}\left(\mathbb{R}^{n}\right)} \leq c_{n}\|g\|_{H^{s}(\mathbb{R})},
$$

where $\langle x\rangle:=1+|x|$ and $\|\cdot\|_{H^{s}}$ denotes the usual Sobolev norm. 
Remark 3.2. It is known that (9) holds whenever $V \geq 0$ is locally integrable.

Proposition 3.3. Let $\alpha=0,1$. Suppose $V \geq 0$ and $e^{-t H}$ satisfies the upper Gaussian bound

$$
\left|\nabla_{x}^{\alpha} e^{-t H}(x, y)\right| \leq c_{n} t^{-(n+\alpha) / 2} e^{-c|x-y|^{2} / t}, \quad \forall t>0 .
$$

If $\left\{\varphi_{j}\right\}_{j \in \mathbb{Z}}$ is a dyadic system satisfying (i), (ii), then for each $N \geq 0$

$$
\left|\nabla_{x}^{\alpha} \varphi_{j}(H)(x, y)\right| \leq c_{N} 2^{j(n+\alpha) / 2}\left(1+2^{j / 2}|x-y|\right)^{-N}, \quad \forall j .
$$

Proof. Write

$$
\nabla_{x}^{\alpha} \varphi_{j}(H)(x, y)=\int_{z} \nabla_{x}^{\alpha} e^{-t H}(x, z)\left(e^{t H} \varphi_{j}(H)\right)(z, y) d z
$$

By (10) we have

$$
\begin{gathered}
\left|\nabla_{x}^{\alpha} \varphi_{j}(H)(x, y)\right| \\
\leq c_{n} t^{-(n+\alpha) / 2} \int e^{-c|x-z|^{2} / t}\langle(x-z) / \sqrt{t}\rangle^{N}\langle(x-z) / \sqrt{t}\rangle^{-N}\langle(z-y) / \sqrt{t}\rangle^{-N} \\
\cdot\langle(z-y) / \sqrt{t}\rangle^{N}\left|\left(e^{t H} \varphi_{j}(H)\right)(z, y)\right| d z \\
\leq c_{n} t^{-(n+\alpha) / 2}\langle(x-y) / \sqrt{t}\rangle^{-N} \int\langle(z-y) / \sqrt{t}\rangle^{N}\left|\left(e^{t H} \varphi_{j}(H)\right)(z, y)\right| d z .
\end{gathered}
$$

Setting $t=t_{j}:=2^{-j}$, we see that $g_{j}(x):=e^{t_{j} x} \varphi_{j}(x)$ also satisfies conditions (i), (ii). Writing $g_{j}(x)=g_{0}\left(2^{-j} x\right)$, then supp $g_{0} \subset\left\{\frac{1}{4} \leq\right.$ $|x| \leq 1\}$ and

$$
\left\|g_{0}\right\|_{H^{N}(\mathbb{R})} \leq\left\|g_{j}\left(2^{j} x\right)\right\|_{C^{N}(\mathbb{R})} \leq c_{N} .
$$

Thus an application of Lemma 3.1 with $g=g_{0}, \beta=N$ proves the proposition.

3.4. Hermite operator $H=-\Delta+|x|^{2}$. To verifies Assumption 1.1 it is sufficient to show $e^{-t H}$ satisfies the upper Gaussian bound in (10), according to Proposition 3.3 .

For $k \in \mathbb{N}_{0}$, let $h_{k}$ be the $k^{t h}$ Hermite function with $\left\|h_{k}\right\|_{L_{2}(\mathbb{R})}=1$ such that

$$
\left(-\frac{d^{2}}{d x^{2}}+x^{2}\right) h_{k}=(2 k+1) h_{k} .
$$

Then $\left\{h_{k}\right\}_{0}^{\infty}$ forms a complete orthonormal system (ONS) in $L_{2}(\mathbb{R})$. In $L_{2}\left(\mathbb{R}^{n}\right)$, the ONS is given by $\Phi_{k}(x):=h_{k_{1}} \otimes \cdots \otimes h_{k_{n}}, k=\left(k_{1}, \ldots, k_{n}\right) \in$ $\mathbb{N}_{0}^{n}$. 
By Mehler's formula [18, Ch.4] or [19, the heat kernel has the expression

$$
\begin{aligned}
e^{-t H}(x, y) & =\sum_{k \in \mathbb{N}_{0}^{n}} e^{-t(n+2|k|)} \Phi_{k}(x) \Phi_{k}(y) \\
& =\frac{1}{(2 \pi \sinh (2 t))^{n / 2}} e^{-\frac{1}{2} \operatorname{coth}(2 t)\left(|x|^{2}+|y|^{2}\right)+\operatorname{cosech}(2 \mathrm{t}) x \cdot y}
\end{aligned}
$$

for all $t>0, x, y \in \mathbb{R}^{n}$.

It is easy to calculate to find that there exist constants $c, c^{\prime}>0$, $0<c_{0}, c_{1}, c_{0}^{\prime}, c_{1}^{\prime}<1$ and $t_{0}>1$ such that

$$
\begin{gathered}
p_{\frac{t}{2}}(x, y) \leq c \begin{cases}t^{-n / 2} e^{-c_{0}|x-y|^{2} / t} & t \leq t_{0} \\
e^{-n t / 2} e^{-c_{1}|x-y|^{2}} & t>t_{0}\end{cases} \\
\left|\nabla_{x} p_{\frac{t}{2}}(x, y)\right| \leq c^{\prime} \begin{cases}t^{-(n+1) / 2} e^{-c_{0}^{\prime}|x-y|^{2} / t} & t \leq t_{0} \\
e^{-n t / 2} e^{-c_{1}^{\prime}|x-y|^{2}} & t>t_{0},\end{cases}
\end{gathered}
$$

where $p_{t}(x, y):=e^{-t H}(x, y)$. Hence (10) holds.

Remark 3.5. For the Hermite operator, the decay estimates similar to (1), (2) were previously obtained in [10] in one dimension and 6] in ndimension. The latter used Heisenberg group method. Proposition 3.3 shows that using heat kernel estimate we can obtain a simpler proof.

Remark 3.6. When $V$ is negative, the heat kernel estimate (9) is not available, espcecially in low dimensions $n=1,2$. but Assumption 1.1 still holds in the high energy case $(j \geq 0)$ for certain short range potentials. A special example is the one dimensional Pöschl-Teller model $V(x)=-\nu(\nu+1) \operatorname{sech}^{2} x, \nu \in \mathbb{N}$, cf. 14. We will discuss the problem in more detail in [23] where $V$ is assumed to have only polynomial decay at infinity.

\section{REFERENCES}

[1] J. Benedetto, S. Zheng, Besov spaces for the Schrödinger operator with barrier potential (submitted). http://lanl.arXiv.org/math.CA/0411348. (2005).

[2] P. D'Ancona, V. Pierfelice, On the wave equation with a large rough potential, J. Funct. Anal. 227 (2005), no. 1, 30-77.

[3] E. Davies, Heat Kernels and Spectral Theory, Cambridge University Press, Cambridge, 1989.

[4] _ Pointwise bounds on the space and time derivatives of heat kernels, J. Operator Theory 21 (1989), 367-378.

[5] X. Duong, L. Yan, Duality of Hardy and BMO spaces associated with operators with heat kernel bounds, J. Amer. Math. Soc. 18 (2005), 943-973.

[6] J. Dziubańsk, Triebel-Lizorkin spaces associated with Laguerre and Hermite expansions, Proc. Amer. Math. Soc. 125 (1997), 3547-3554. 
[7] J. Dziubański, J. Zienkiewicz, Hardy spaces $H^{1}$ for Schrödinger operators with compactly supported potentials, Ann. Mat. Pura Appl. (4) 184 (2005), no. 3, $315-326$.

[8] J. Dziubański and J. Zienkiewicz, $H^{p}$ spaces for Schrödinger operators, in: Fourier Analysis and Related Topics, Banach Center Publ. 56, Inst. Math., Polish Acad. Sci., 2002, 45-53.

[9] J. Epperson, Triebel-Lizorkin spaces for Hermite expansions, Studia Math. 114 (1995), 87-103.

[10] _ Hermite and Laguerre wave packet expansions, Studia Math. 126 (1997), no. 3, 199-217.

[11] W. Hebisch, A multiplier theorem for Schrödinger operators, Colloq. Math. 60/61 (1990), no. 2, 659-664.

[12] A. Jensen, S. Nakamura, Mapping properties of functions of Schrödinger operators between $L^{p}$ spaces and Besov spaces, in Spectral and Scattering Theory and Applications, Advanced Studies in Pure Math. 23, 1994.

[13] M. Keel, T. Tao, Endpoint Strichartz estimates, Amer. J. Math. 120 (1998), 955-980.

[14] G. Ólafsson, S. Zheng, Function spaces associated with Schrödinger operators: the Pöschl-Teller potential (submitted).

[15] I. Rodnianski, T. Tao, Long-time decay estimates for the Schrödinger equation on manifolds. Preprint.

[16] W. Schlag, A remark on Littlewood-Paley theory for the distorted Fourier transform, http://lanl.arXiv.org/math.AP/0508577, (2005).

[17] B. Simon, Schrödinger semigroups, Bull. Amer. Math. Soc. 7 (1982) no.3, 447526.

[18] S. Thangavelu, Lectures on Hermite and Laguerre expansions, Princeton Univ. Press, 1993.

[19] Preprint.

[20] H. Triebel, Theory of Function Spaces, Birkhäuser Verlag, 1983.

[21] —, Theory of Function Spaces II, Monographs Math. 84, Birkhäuser, Basel, 1992.

[22] S. Zheng, A representation formula related to Schrödinger operators, Anal. Theo. Appl. 20 (2004), no.3., 294-296.

[23] _ Spectral multipliers, function spaces and dispersive estimates for Schrödinger operators. Preprint.

Department of Mathematics, University of South Carolina, Columbia, SC 29208

E-mail address: shijun@math.sc.edu

URL: http://www.math.sc.edu/ shijun 